Proceedings of the 2006 Winter Simulation Conference

L. F. Perrone, F. P. Wieland, J. Liu, B. G. Lawson, D. M. Nicol, and R. M. Fujimoto, eds.

\title{
EVALUATING ARRIVAL RATE UNCERTAINTY IN CALL CENTERS
}

Thomas R. Robbins

Supply Chain and Information Systems
Penn State University
University Park, PA 16802, U.S.A.

\author{
D. J. Medeiros \\ Paul Dum \\ Industrial \& Manufacturing Engineering \\ Penn State University \\ University Park, PA 16802, U.S.A.
}

\begin{abstract}
Inbound call center operations are challenging to manage, in large part because there is considerable uncertainty in estimates of arrival rates, which vary over time. We have developed a general purpose simulation model for inbound call center operations which supports time varying and uncertain arrival rates along with variable staffing. We outline the conceptual and technical design of the simulation model. We then define and conduct an initial experiment that uses the model to evaluate the impact of arrival rate uncertainty on call center performance. We find that arrival rate uncertainty creates significant planning challenges for managers attempting to satisfy tight performance targets, particularly one-sided performance measures. We also find that abandonment rate has a major impact on call center performance.
\end{abstract}

\section{INTRODUCTION}

Call centers are a large and growing component of the U.S. and world economy (Gans et al. 2003). In 1999 an estimated 1.5 million workers were employed in call centers in the US alone. Large scale call centers are technically and managerially sophisticated operations and have been the subject of substantial academic research.

A call center is a facility designed to support the delivery of some interactive service via telephone communications, typically an office space with multiple workstations manned by agents who place and receive calls (Gans et al. 2003). Call center applications include tele-marketing, customer service, help desk support, and emergency dispatch.

Most call centers support both inbound and outbound operations. An inbound operation takes customer initiated calls while an outbound operation places calls to customers. Inbound and outbound calls are often handled by separate teams of agents, though in some call centers inbound and outbound calls are mixed in a process known as blending.
Even a moderately sophisticated call center is equipped with advanced computer and telecommunications equipment. An inbound call typically connects from the public service telephone network (PSTN) to the call center's switch, the private branch exchange (PBX), over a number of owned or leased trunk lines. Callers may initially connect to an Interactive Voice Response Unit (IVR) where the caller can use her keypad to select options and potentially provide data input to call center system. When callers need to speak to an agent, the call is handled by the Automated Call Distributor (ACD). The ACD routes calls internal to the call center and is responsible for monitoring agent status, collecting data, managing on hold queues, and making potentially complex routing decisions. For example, in call centers that employ skills based routing, a complex decision process is used to match callers and agents based on multiple criteria concerning both the callers and the agents. In addition to the telephone system, a call center agent usually has a computer terminal connected into one or more enterprise applications; these are typically classified under the general category of Customer Relationship Management (CRM).

In lower volume, less sophisticated call centers the telecommunications systems and information processing systems are completely separate, but most moderately sophisticated centers use some form of Computer Telephony Integration (CTI). CTI allows information to pass back and forth between the two systems. The call center system typically has some form of CTI middleware to manage the integration of the telephony and computing systems. With that data the CTI system can orchestrate a screen pop, the simultaneous delivery of a call to an agent's telephone and a screen of information to the same agent's workstation.

Gans et al. (2003) document a series of industry standard measures used to evaluate call center performance. We summarize the key measures here:

- Excess Capacity: the minimum staff level required to provide queue stability is well known to be $N=R_{i}$, where $R_{i}$ is the offer load; the ratio of 
average arrival rate and service rate. $(\mathrm{R}=\lambda / \mu)$. Excess capacity (denoted as $\Delta$ ) is defined as the number of additional agents staffed above the minimum level. $(\Delta=\mathrm{N}-\lambda / \mu)$

- Average Speed of Answer (ASA): this is the average time callers spend on hold, waiting for an agent.

- Telephone Service Factor (TSF): also called the service level, TSA is the fraction of calls for which the delay is below a specified level. For example, a call center may report the TSF as the percent of callers on hold less then 30 seconds.

- Abandonment \%: callers that are put on hold and hang up while in queue are said to have abandoned the system. The proportion of all calls that abandoned is known as the abandonment $\%$ and is a key metric in most call centers.

The paper also outlines a set of call center regimes: three basic categories that describe the staffing/customer service objectives of the call center.

- Quality Driven Regime: customer waiting costs are assumed to dominate the cost of capacity and the objective is to serve the majority of customers without delay. Staffing levels are increased linearly with offered load. Average utilization in this regime is typically low, on the order of $65-75 \%$, and average customer wait time is also low.

- Efficiency Driven Regime: staffing costs are assumed to dominate the cost of customer delay and the operational objective in this regime is to maximize the utilization of the staff.

- Quality Efficiency Driven (QED): an operational environment that attempts to strike some balance between efficiency and customer service. Unlike the quality regime where the fraction of delayed customer is near zero, or the efficiency regime where the fraction delayed is near one, the QED regime balances costs and attempts to achieve some steady delay proportion between 0 and 1 .

\section{CALL CENTER RESEARCH}

Much of the call center research is oriented toward queuing behavior in general as well as the relationship between queuing behavior and staff level decisions. (Halfin and Whitt 1981; Whitt 1989; Whitt 1992a; Whitt 1992b; Borst et al. 2004; Whitt 2005). Another series of papers addresses the specific issues related to staff selection given schedule constraints Many of these papers are motivated by call center applications, but several are applicable to the staffing problem at any operation. The general problem presented in these papers is developing a staff schedule that meets a time varying set of agent requirements. These models generally assume the call center staffing requirements are known with certainty in each 15 or 30 minute time period. The models also assume that agents may be scheduled to a finite set of scheduled start and end times. The more advanced models include an explicit break schedule. These models are extensions of the general set covering algorithm, and when breaks are scheduled explicitly the problem becomes NP hard (Garey and Johnson 1979). Given the intractability of the scheduling problem many researchers have sought heuristic scheduling solutions. A large set of papers addresses this problem (Bechtold and Jacobs 1990; Brusco and Johns 1996; Brusco and Jacobs 1998; Brusco and Jacobs 2000). As mentioned previously these period-by-period models assume agent requirements can be defined explicitly. The implicit assumption is that the queue achieves equilibrium in each time period and that arrival rates are known with a high degree of confidence. This breaks the optimization problem into two components: a server sizing component done exogenously to the model, and a schedule optimization component. We know of one paper that breaks with this approach. Koole and Sluis (2003) attempt to develop a staffing model that optimizes a global objective, i.e. an average performance metric over a longer time period.

Much of the academic literature on call centers, and queuing systems in general, is done using closed form analytical equations, using approximations as necessary. The benefits from closed form analysis are substantial; equations that describe system behavior, even when approximate, are easily generalized and applied to other related problems. Management insight is often easier to develop from closed form expressions that describe system behavior.

However there are some serious limitations in terms of the results that closed form analysis can provide. Analytical models are usually derived given the following set of assumptions - see (Gans et al. 2003) for a more detailed discussion.

- Poisson Processes: interarrival and service times are typically assumed to be exponentially distributed so that some form of an Erlang model can be used. The assumption of exponentially distributed arrivals is theoretically supported and empirically verified (Brown et al. 2005). The same study, however, finds that talk time is not distributed exponentially but rather exhibits a lognormal distribution.

- Independence: interarrival and service times are virtually always assumed to be independent of each other. Brown et al. (2005) also find positive correlation between service time and arrival rate so that calls are longest during the busiest time of the day. 
- Steady State: arrival rates are often considered to be time invariant as is staffing. Models in which rates or staffing are variable generally assume each is constant in a time bucket (say 15 or 30 minutes). Stationary measures are calculated in the interval, implicitly assuming the system quickly obtains stability. Analysis of transient behavior is limited, but in reality call centers subject to highly variable arrival rates may rarely achieve steady state.

- Arrival rate uncertainty: the interarrival time of calls is recognized as uncertain and highly variable, being described in most models by an exponential distribution. The arrival rate $\lambda$, is however usually assumed to be known. In reality call centers are staffed based on expected arrival rates which may suffer from high levels of uncertainty. Limited research has been done to explore the effect of arrival rate uncertainty.

- Routing: most standard models assume a simple routing of calls. In reality call centers often have more complex routings. Calls may be conditionally routed based on priority or the skills required to service the call type: so called skills based routing.

While much of the theoretical analysis of call centers ignores the issue of abandonment, abandonment is discussed in detail in Mandelbaum and Zeltyn (2004). This paper analyzes call centers in the context of the Erlang-A model, an extension of the basic Erlang-C $(\mathrm{M} / \mathrm{M} / \mathrm{N})$ model that assigns an exponentially distributed patience factor to callers. A caller abandons the system when his wait time exceeds his patience. A call center staffed so that is unstable in an Erlang-C model is shown to be stable, and in some situations to exhibit desirable performance characteristics. Stated another way, significant cost savings can be achieved if the call center is willing to accept moderate levels of customer abandonment. A key limitation of the Erlang-A model is the assumption of exponential patience.

In addition to the large body of theoretical/analytic models addressing call centers, we know of 2 papers that explicitly analyze the statistical data generated in a call center. Mandelbaum et al. (2000) and Brown et al. (2005) provide a statistical analysis of the same set of data gathered from a bank's call center. They find that call time has a lognormal distribution; in contrast most analytical work makes the simplifying assumption that talk time is exponential. The authors also find that the hazard rate for abandonment (the time phased probability for abandoning) is bimodal. A large peak occurs a few seconds after the caller realizes he must wait, while a second peak occurs 60 seconds later after a please wait message is played. In the tail, the hazard rate becomes approximately constant, sup- porting the concept of exponential patience for those willing to wait at least a moderate time.

In addition, a few papers have been written on call center operations in practice. A series of papers discusses call center operations at L. L. Bean (Andrews and Parsons 1989; Quinn et al. 1991; Andrews and Parsons 1993; Andrews and Cunningham 1995). The authors discuss issues related to forecasting, resource allocation and scheduling. The theme that runs through these papers is the challenge related to determining the appropriate number of agents to staff given the tradeoff of operational costs and customer service. A practice paper that highlights the use of simulation in call center planning is Saltzman and Mehrotra (2001). In this paper the authors document the use of a call center simulation model to help a software company determine approximate staff level requirements for a new service offering.

\section{CALL CENTER MODEL}

Our first effort was to develop a general purpose inbound call center simulation model. Our model assumes infinite trunk capacity and homogeneous agents, and has the following characteristics:

- Time varying arrival rate: the system supports exponential arrival rates that may vary by $15 \mathrm{~min}$ ute time periods. The arrival rates are read from an external data file.

- Arrival Rate Error: the model further allows for the specification of an arrival rate error so that the realized arrival rate in each 15 minute period is $\lambda_{r}=\bar{\lambda}+\varepsilon_{a}$ where $\bar{\lambda}$ is the arrival rate specified and $\varepsilon_{\mathrm{a}}$ is a normally distributed error with mean $\mu_{\mathrm{a}}$ and standard deviation $\sigma_{\mathrm{a}}$. Note that setting $\mu_{\mathrm{a}}$ and $\sigma_{\mathrm{a}}$ to zero reduces the model to a known arrival rate.

- Staffing Levels: the staffing level is configurable in 15 minute periods, and is read from an external file.

- Service Time Distribution: the model allows service time to be modeled as either exponential or lognormal.

- Abandonment: we recognize from the empirical research that abandonment may be bi-modal so the system models abandonment as follows:

- Each caller is willing to wait with probability $\mathrm{p}_{\mathrm{w}}$, and unwilling to wait with probability 1 $\mathrm{p}_{\mathrm{w}}$. If the caller is unwilling to wait he abandons immediately if put on hold. The parameter $p_{w}$ is a global parameter for the model and may be specified externally.

- Callers who are willing to wait have a patience that is uniformly distributed on 
$\left[\mathrm{w}_{\mathrm{a}}, \mathrm{w}_{\mathrm{b}}\right]$. A caller will wait up until his patience and if not served by then will abandon. The wait parameters are global and externally specified.

- Operation Time: the call center may be a continuous $(24 \times 7)$ operation or may have a finite operating time. In the latter case, calls are blocked at the end of the shift but any calls in process or in queue are completed.

- Statistics: the model collects and reports on the following statistics: call volume, abandonment rate, agent utilization, queue size, ASA, calls with zero wait time, TSF, and maximum hold time. For each statistic a file is created that stores the value of the statistic for each hour of simulated time.

Modeling issues included constructing a model that was entirely data driven, providing for external analysis of the hour-by-hour statistics, modeling the nonstationary arrival process, and incorporating abandonment. Our model uses external data files for input of variable rates and output of hourly statistics, while flags are used to control items such as enabling hourly logging, abandonment, variable rates, etc. We plan to replace the data files with an ActiveX interface for ease of use. Henderson (2005) provides an excellent discussion of alternatives for modeling arrival processes in call centers; our model implements a nonhomogeneous Poisson process. To model abandonment, the arriving entity is cloned; the clone waits for the (random) patience time and removes its original entity from the list of calls on hold if that entity is still on the list.

\section{ANALYSIS OF FORECASTING ERROR}

The model described above is a general purpose call center simulation that can be applied to the analysis of a wide range of scenarios. We conduct an experiment to examine the degradation in system performance when arrival rates vary from expectation. We define a scenario with a 12 hour shift, from $8 \mathrm{AM}$ to $8 \mathrm{PM}$. Call volume in each 15 minute period is given by $\lambda_{\mathrm{a}}=\lambda_{\mathrm{a}}+\varepsilon_{\mathrm{a}}$, where $\lambda_{\mathrm{a}}=1,000$ calls $/ \mathrm{hr}$. and $\varepsilon_{\mathrm{a}} \sim \mathrm{N}\left(\mu_{\mathrm{a}}, \sigma_{\mathrm{a}}\right)$. Service time is lognormally distributed with an expected talk time of 10 minutes and a standard deviation of 2 minutes. Callers are willing to wait with probability $\mathrm{p}_{\mathrm{w}}=95 \%$. Callers put on hold have a patience uniformly distributed between 60 and 300 seconds. We wish to explore effects of arrival rate uncertainty so our experiment varies $\mu_{\mathrm{a}}$ from -200 to 200 and $\sigma_{\mathrm{a}}$ from 0 to 200.

Since our objective is to investigate how different call center regimes respond to arrival rate forecasting errors, we assume we have 3 call centers with the following staffing objectives.
- Call Center 1: call center 1 is efficiency driven. Staffing is planned so as to achieve a $96 \%$ agent utilization rate.

- Call Center 2: call center 2 is quality driven. Staffing is planned so that the $99 \%$ of customers will have a wait time of less then 3 seconds.

- Call Center 3: call center 3 utilizes a quality efficiency driven policy. Staffing is planned so that the expected wait time for a customer is less then 2 seconds and abandonment rate is less then $5 \%$.

\subsection{Baseline Analysis}

The first step in this analysis is to find the baseline staffing level for each of the 3 scenarios. To do this we conduct a numerical experiment where we vary the staff levels and calculate the relevant performance metrics. We varied staff levels from 125 to 250 in increments of 1 . We conducted our experiment with a warmup period of 4 hours, an 8 hour analysis period and 5 runs per staff level. This experiment required 630 runs and executed in about 45 minutes in the background. Based on this analysis we are able to determine the baseline staffing for each of the call centers in our experiment; call center 1: 160 agents, call center 2: 194 agents, call center 3: 170 agents.

\subsection{Variability Analysis}

In order to test the impact of variability in arrival rate we set up an experiment in which we vary several factors:

- Estimate Error Mean: the error mean is varied from -200 to 200 in increments of 100.

- Estimate Error Standard Deviation: the error standard deviation is set to 4 discrete values, 0 , 10, 100, 200.

- Staff Level: staffing is set to the 3 discrete levels selected from the baseline analysis: 160, 170, and 194.

We run the model with a 4 hour warmup period and an 8 hour snap length. We run 20 iterations at each point resulting in 1,200 total runs. The results for each regime are discussed below.

\subsubsection{Efficiency Driven Regime}

In the efficiency driven call center the key management metric is utilization. The staffing goal is to keep utilization levels high, in our example at 96\%. Figure 1 shows the results of the simulation analysis performed to vary arrival rate. 


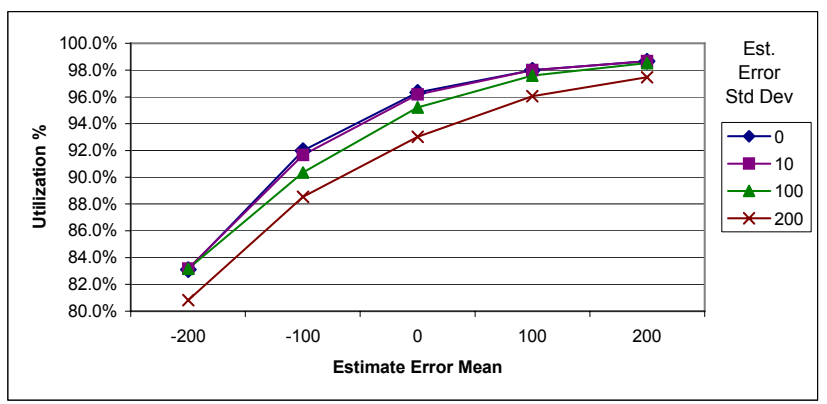

Figure 1: Effect of Arrival Rate Uncertainty on Utilization for the Efficiency Driven Regime

We make the following observations:

- A small standard deviation has little impact. (i.e. if the arrival rate varies from its mean over the course of the day by a small amount the impact is minimal.)

- Larger standard deviations tend to decrease utilization since utilization will drop during the slow periods and abandonment will rise during the busy periods.

- Positive bias (i.e. underestimating the arrival rate) has a limited effect. Since target utilization is very high, a higher call rate creates only a small increase in utilization.

- Negative bias adversely affects the utilization statistic. A $10 \%$ overestimation of arrival rate causes utilization to drop by $4 \%$. A $20 \%$ error causes utilization to drop by $12 \%$, and if this is accompanied by arrival rate variability during the day, utilization may drop below $82 \%$.

In addition to the management objective of high utilization we also wish to see the impact on customer service metrics. For this analysis we chose to look at abandonment rate, as shown in Figure 2.

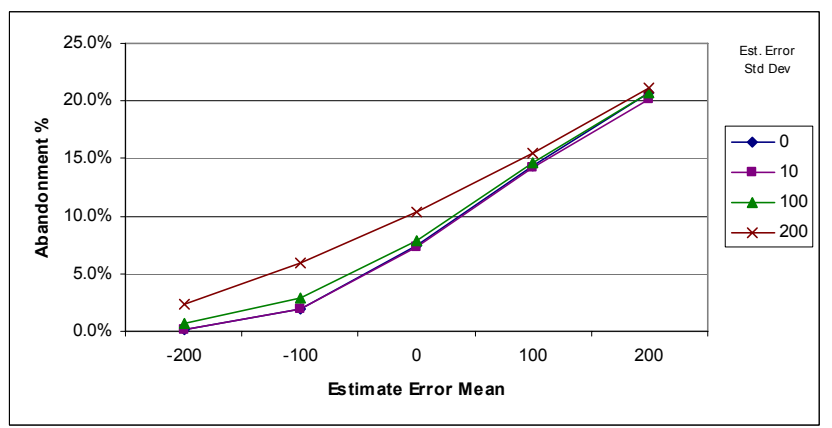

Figure 2: Effect of Arrival Rate Uncertainty on Abandonment for the Efficiency Driven Regime

We make the following observations concerning abandonment rate:
- Arrival rate variability drives abandonment rate up.

- Call volumes above forecast will significantly increase abandonment rate from its base level of about $8 \%$ to over $20 \%$.

- Arrival rates below expectation will decrease abandonment rate substantially.

The key conclusions from this analysis are that the performance metrics in an efficiency driven call center are very sensitive to the estimated arrival rate, and that maintaining a high utilization rate is very difficult in the presence of customer abandonment. Given the large decline in utilization for below expected arrival rates, a call center manager measured on staff utilization is likely to estimate call volume very conservatively (i.e. systematically underestimate call volume). With utilizations slightly above target, abandonment rates are likely to be large.

\subsubsection{Quality Driven Regime}

In the quality driven regime the key management objective is to maintain a specified percentage of customers whose wait time is less then an established target. Figure 3 shows the percentage of customers who achieve the target wait level as a function of the arrival rate parameters. Recall that the target level is very high, $99 \%$ with a wait time of 3 seconds or less.

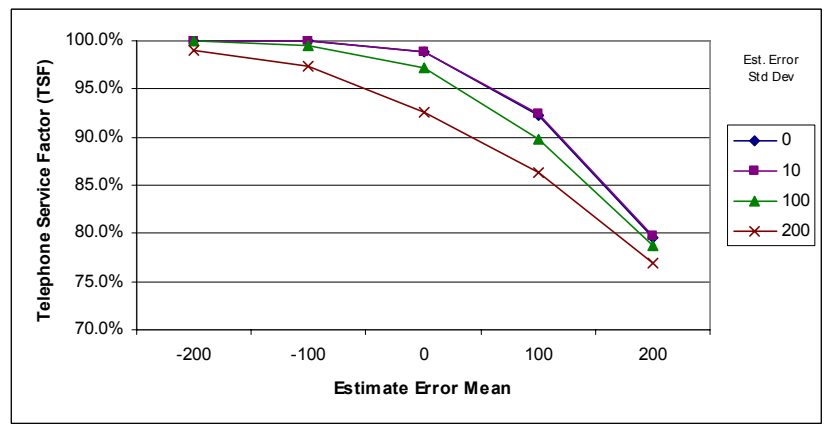

Figure 3: Effect of Arrival Rate Uncertainty on Calls with Low Wait for the Quality Driven Regime

Summary observations are:

- Again, small variations in the arrival rate standard deviation have limited impact.

- High variability in arrival rate affects the performance metric significantly. If the daily arrival rate is on target, but varies significantly during the day, the call center misses the $99 \%$ target by over $5 \%$.

- Underestimating the arrival rate has a major negative impact. A call volume $10 \%$ greater than ex- 
pected can decrease the TSF by $8 \%$, and a $20 \%$ forecast error drops the TSF to below $78 \%$.

We also examine the abandonment rate in this regime. Given the high percentage of customers to be served in less then 2 seconds the implied target for abandonment rate is very low. Figure 4 shows the impact of variable arrival rate.

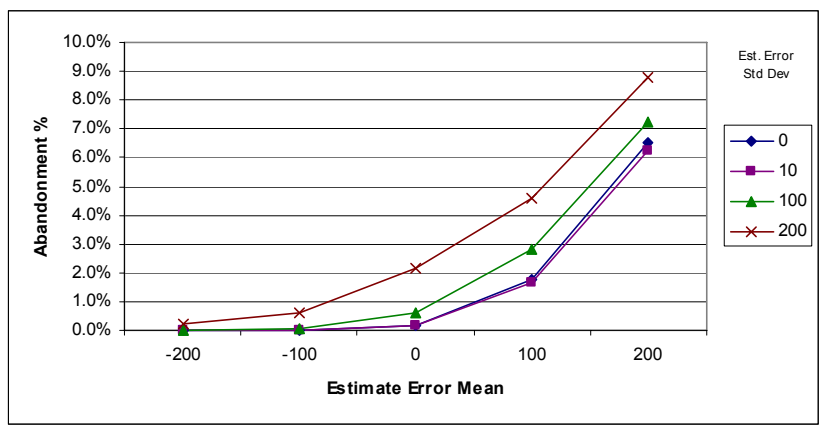

Figure 4: Effect of Arrival Rate Uncertainty on Abandonment $\%$ for the Quality Regime

Summary observations for this case include:

- The abandonment rate is highly sensitive to arrival rate variability and bias.

- Because the model assumes a substantial portion of customers will abandon immediately when put on hold, small increases in arrival rate can have major impacts on abandonment rate.

\subsubsection{Quality-Efficiency Driven Regime}

In the quality-efficiency driven regime the key management objective is to maintain a specified expected wait time. Unlike the other regimes, this implicitly makes a tradeoff between agent time and customer wait time. Recall that the target level for our call center is an average wait time of 2 seconds. The expected wait time is shown in Figure 5.

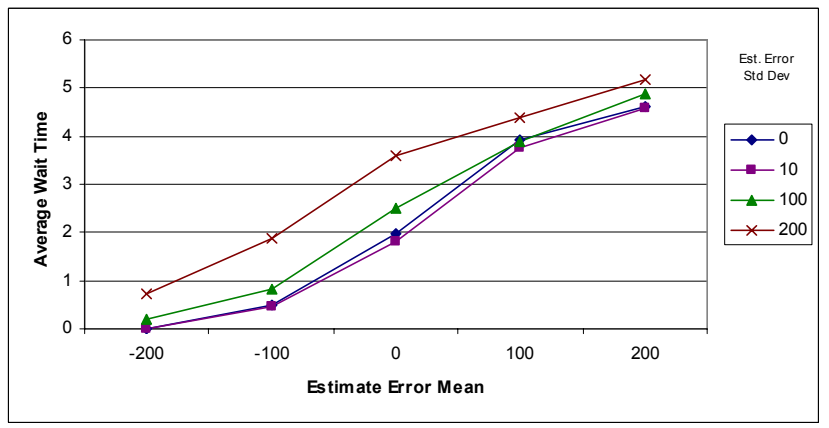

Figure 5: Effect of Arrival Rate Uncertainty on Wait Time for the Quality-Efficiency Driven Regime
Summary observations are:

- Again, small variations in the arrival rate standard deviation have limited impact.

- Larger variations in the period to period arrival rate increase wait time.

- A bias in the overall arrival rate has a roughly near linear impact on expected wait time. A $20 \%$ increase in call volume causes wait time to roughly double. A $20 \%$ decrease in call volume causes wait time to fall near zero.

Once again we also examine the impact on customer service as measured by abandonment rate (see Figure 6).

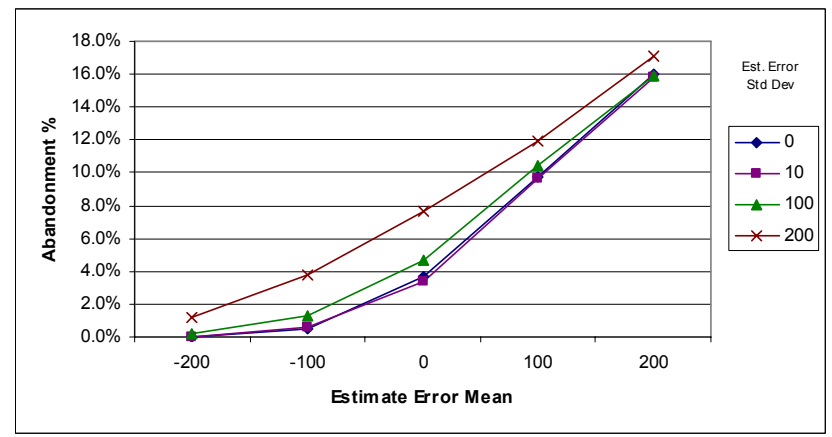

Figure 6: Effect of Arrival Rate Uncertainty on Abandonment for the Quality-Efficiency Driven Regime

We observe the following effects:

- Again we see that deviations in call volume have a major impact on abandonment rate. Increased call rates cause abandonment rates to rise significantly.

- Overall the performance metrics for this regime tend to behave a bit more linearly then for the previous two regimes. Abandonment percentage is increasing at a faster than linear rate, but lies between the performance of the other regimes.

\section{SUMMARY AND CONCLUSIONS}

We have analyzed the effect of call arrival rate uncertainty across call center regimes in an admittedly narrow experiment. A more thorough analysis would have examined the impact of variability at various call center scales (small, medium, and large centers) and across a range of efficiency and quality parameters. However, our analysis does serve its intended purpose: to show that the various call center staffing models are highly sensitive to the arrival rate assumptions.

In our analysis we allowed call volume to vary from expectation and showed that performance levels differ significantly from target when arrival rate varies from plan. Empirical research has shown that call arrival rates are 
highly variable and difficult to predict (Brown et al. 2005). Furthermore, a qualitative analysis of the incentives in the quality and efficiency regimes illustrates that management incentives to meet one sided goals may cause rational managers to systematically bias arrival rate estimates as a cushion.

The quality-efficiency paradigm, on the other hand, is a two sided objective: the goal is to achieve a specific level of performance, rather then be better then some threshold. We argue that this type of paradigm, where deviation from plan above or below is equally costly, does not create incentives for an estimation bias. The performance metric in the quality-efficiency example is also slightly less sensitive to disruptions than the pure efficiency or pure quality metrics.

Overall we find that the high volume call center we examined in this analysis is highly sensitive to arrival rate estimation. We expect that lower volume centers, with lower economies of scale, would suffer equal or greater disruption from shifts in call volume. Since arrival rates have been shown to be highly variable, we argue that theoretical models that assume a known arrival rate are suspect, yet this is common practice. We believe models that explicitly recognize the variability in the arrival rate, while more complex to solve, would provide better results.

Our study has also demonstrated some of the limitations of a simulation based analysis. Because this analysis does not develop closed form results we must test across a wide range of input parameter values. The admittedly narrow analysis we report in this paper required thousands of simulation runs. To demonstrate that the general findings are applicable for different sized call centers, or differently specified target metrics, requires either faith, or many more simulation runs.

The model we have developed is very general and may be used to analyze various issues in call center performance. Our ongoing analysis will continue to evaluate the impact of uncertainty in system parameters. In particular we seek to investigate the equilibrium assumption when arrival rates are highly variable through the course of the day.

\section{REFERENCES}

Andrews, B. and H. Parsons. 1993. Establishing Telephone-Agent Staffing Levels through Economic Optimization. Interfaces 23(2) 14.

Andrews, B. H. and S. M. Cunningham. 1995. L.L. Bean Improves Call-Center Forecasting. Interfaces 25(6) 1.

Andrews, B. H. and H. L. Parsons. 1989. L.L. Bean Chooses a Telephone Agent Scheduling System. Interfaces 19(6) 1.

Bechtold, S. E. and L. W. Jacobs. 1990. Implicit Modeling of Flexible Break Assignments in Optimal Shift Scheduling. Management Science 36(11) 1339-1351.
Borst, S., A. Mandelbaum, and M. Reiman. 2004. Dimensioning Large Call Centers. Operations Research 52(1) 17-35.

Brown, L., N. Gans, A. Mandelbaum, A. Sakov, H. Shen, S. Zeltyn, and L. Zhao. 2005. Statistical Analysis of a Telephone Call Center: A Queueing-Science Perspective. Journal of the American Statistical Association 100(469) 36-50.

Brusco, M. J. and L. W. Jacobs. 1998. Personnel Tour Scheduling When Starting-Time Restrictions Are Present. Management Science 44(4) 534-547.

Brusco, M. J. and L. W. Jacobs. 2000. Optimal Models for Meal-Break and Start-Time Flexibility in Continuous Tour Scheduling. Management Science 46(12) 16301641.

Brusco, M. J. and T. R. Johns. 1996. A sequential integer programming method for discontinuous labor tour scheduling. European Journal of Operational Research 95(3) 537-548.

Gans, N., G. Koole, and A. Mandelbaum. 2003. Telephone call centers: Tutorial, review, and research prospects. Manufacturing \& Service Operations Management 5(2) 79-141.

Garey, M. R. and D. S. Johnson. 1979. Computers and intractability: a guide to the theory of NP-completeness, W. H. Freeman. San Francisco.

Halfin, S. and W. Whitt. 1981. Heavy-Traffic Limits for Queues with Many Exponential Servers. Operations Research 29(3) 567-588.

Henderson, S. 2005. Should we model dependence and nonstationarity, and if so how? In Proceedings of the 2005 Winter Simulation Conference, ed. M. E. Kuhl, N. M. Steiger, F. B. Armstrong, and J. A. Joines, 120129. Piscataway, New Jersey: Institute of Electrical and Electronics Engineers.

Jennings, O. B. and A. Mandelbaum. 1996. Server staffing to meet time-varying demand. Management Science 42(10) 1383.

Koole, G. and E. van der Sluis. 2003. Optimal shift scheduling with a global service level constraint. IIE Transactions 35 1049-1055.

Mandelbaum, A. and S. Zeltyn. 2005. Service Engineering in Action: The Palm/Erlang-A Queue, with Applications to Call Centers. Working Paper [online] 29p. Available via <iew3.technion.ac.il/serv eng/Refeences/references.html> [accessed March 10, 2006].

Mandelbaum A., Sakov A., and Zeltyn S. 2000. Empirical Analysis of a Call Center. Technical Report [online] 73p. Available via <iew3.technion.ac.il/ serveng/References / references.html> [accessed March 10, 2006].

Quinn, P., B. Andrews, and H. Parsons, 1991. Allocating Telecommunications Resources at L. L. Bean, Inc. Interfaces 21(1) 75. 
Saltzman, R. M. and V. Mehrotra. 2001. A Call Center Uses Simulation to Drive Strategic Change. Interfaces 31(3) 87.

Whitt, W. 1989. An Interpolation Approximation for the Mean Workload in a GI/G/1 Queue. Operations Research 37(6) 936-952.

Whitt, W. 1992a. Asymptotic Formulas for Markov Processes with Applications to Simulation. Operations Research 40(2) 279-291.

Whitt, W. 1992b. Understanding The Efficiency Of MultiServer Service Systems. Management Science 38(5) 708-723.

Whitt, W. 2005. Engineering Solution of a Basic CallCenter Model. Management Science 51(2) 221-235.

\section{AUTHOR BIOGRAPHIES}

THOMAS ROBBINS is currently pursuing a Ph.D. in Business Administration and Operations Research from Penn State University. He worked in professional services for approximately 18 years before entering the $\mathrm{PhD}$ program. He served as a partner at Ernst and Young, and a Vice President at CGEY and Aztec Software. He holds an MBA from Case Western Reserve and a BSEE from Penn State. His research interests include service operations management. His e-mail address is <trr147@psu.edu>.

D. J. MEDEIROS is Associate Professor of Industrial Engineering at Penn State University. She holds a Ph.D. and M.S.I.E from Purdue University and a B.S.I.E. from the University of Massachusetts at Amherst. She has served as track coordinator, Proceedings Editor, and Program Chair for WSC. Her research interests include manufacturing systems control and CAD/CAM. She is a member of IIE and SME. Her e-mail address is $<\mathrm{djm} 3$ @esu . edu $>$.

PAUL DUM graduated from Penn State in May 2006 with an MS in IE and Operations Research; he also received the B.S.I.E from Penn State. His research focused on the application of IE techniques to the healthcare field. He is currently employed in the Operations Directorate of NASA's Johnson Space Center in Houston, Texas. 